\title{
Mechanical dyssynchrony \& CRT: Is it time for guideline updates?
}

\author{
Wael AlJaroudi, MD, FASNC ${ }^{\mathrm{a}}$ \\ a Division of Cardiovascular Medicine, Clemenceau Medical Center, Beirut, Lebanon
}

Received May 23, 2019; accepted May 23, 2019

doi:10.1007/s12350-019-01773-1

\section{See related article, pp. 2174-2184}

Cardiac resynchronization therapy (CRT) improves left ventricular (LV) remodeling, quality of life, and survival among patients with heart failure, reduced ejection fraction (EF) and electrical dyssynchrony. ${ }^{1-3}$ The 2012 guidelines for CRT implantation have very stringent class I indication as they require $\mathrm{EF} \leq 35 \%$, New York Heart Association (NYHA) class $\geq$ II with left bundle branch block (LBBB) and QRS $\geq 150 \mathrm{~ms}$ as compared to a lower QRS threshold $\geq 120-130 \mathrm{~ms}$ with 2008 guidelines. ${ }^{4}$ This was driven in part by the results from the Multicenter Automatic Defibrillator Implantation Trial With Cardiac Resynchronization Therapy (MADIT-CRT) that identified these parameters among others as powerful predictors of response to CRT. ${ }^{5}$ Still, a significant proportion of patients do not derive the expected benefit from such expensive and invasive procedure; and the rate of non-responders has not significantly changed over the last decade despite update in guidelines and more rigorous selection criteria. ${ }^{6,7}$

Extensive research has been done over the last decade to improve patient selection for CRT. One of the aims for CRT is to restore mechanical synchronicity, improving cardiac output and promoting LV reverse remodeling. Hence, significant work on detecting and quantifying indices of LV mechanical dyssynchrony (LVMD) was under way. While many parameters reflecting certain aspects of LVMD with echocardiography were established, none of them were able to

Reprint requests: Wael AlJaroudi, MD, FASNC, Division of Cardiovascular Medicine, Clemenceau Medical Center, Beirut, Lebanon; wjaroudi@hotmail.com

J Nucl Cardiol 2021;28:2185-9.

1071-3581/\$34.00

Copyright (C) 2019 American Society of Nuclear Cardiology. predict response to $\mathrm{CRT}^{8}$; this was in part driven by the high inter and intra-observer variability obtaining these indices. Other imaging modalities such as cardiac magnetic resonance imaging provide a more robust and reproducible LVMD indices; however, the limited availability, high cost and time have restricted its use. ${ }^{9}$

Chen et al published a pioneer paper in 2005 and showed that LVMD indices can be derived retrospectively from previously and routinely performed gated single-photon emission tomography (SPECT) using phase analysis without additional cost or radiation burden; two parameters stood up: phase standard deviation (SD) and histogram bandwidth (BW). ${ }^{10}$ Shortly after, many papers followed and showed several important key findings: (1) LVMD indices by phase analysis are reproducible and repeatable ${ }^{11}$; (2) LVMD correlate mildly to moderately with electrical dyssynchrony, hence being able to discriminate better responder to CRT than QRS duration, ${ }^{12}$ (3) the higher the phase SD/ $\mathrm{BW}$ indices (i.e. the more the LVMD), the greater the response with CRT. ${ }^{13,14}$

Moreover, there were significant efforts to further refine patient selection for CRT. An important lesson was learned: patients with large scar burden respond poorly to CRT, particularly if the LV lead is placed overlying a scarred area. ${ }^{15}$ In addition, placing the LV lead in concordance with the latest site of onset of mechanical contraction provided better response to CRT. ${ }^{16}$ This was made possible by taking advantage of the polar map generated rather than LVMD indices alone. Hence, using these perfusion and contraction polar maps to guide electrophysiologist to place the lead correctly seems crucial and awaits verification in a randomized trial. ${ }^{17}$

The problem with phase SD/BW is that different cut-off values for CRT responders were published. ${ }^{13,14}$ The paper by Tao et al provided an alternative solution; while studying the generated polar maps of mechanical contraction, two contraction patterns were identified: U shape and non-U shape. ${ }^{18}$ Although all patients met the 
guidelines criteria for CRT, only $50 \%$ of those with non$\mathrm{U}$ shape contraction pattern responded at 6 months vs $90 \%$ of those with U shape pattern. After adjustment for QRS, scar and phase SD, U shape contraction pattern remained an independent predictor of CRT response at 6 months (odds ratio $16.0, P=.012$ ). ${ }^{18}$

In this current review paper, the authors summarized the role of LMVD in predicting response to CRT, compared phase analysis to other imaging modalities, and focused on the recent undergoing research, challenges and future of this technology. ${ }^{19}$ While most papers focused on systolic LVMD, the importance of diastolic LVMD rose to the surface and was extensively reviewed and brought to light in the review article. ${ }^{19}$ Indeed, it is well known that diastolic dysfunction plays a crucial role in LV mechanics, impacts symptoms and has significant prognostic value. ${ }^{20}$ Diastolic parameters such as peak filling rate and time to peak filling used to be determined with nuclear imaging as surrogate for diastolic function long before echocardiography introduced tissue Doppler imaging. In a step forward, phase analysis quantifies diastolic LVMD indices by analysis the diastolic part of gated SPECT. ${ }^{21}$ The review paper nicely summarized the recent papers on diastolic LVMD which seems to be complimentary to systolic LVMD, have prognostic value and are likely to play an important role in fine tuning patient selection for CRT. ${ }^{19,22,23}$

It seems things are getting bit more complex with several parameters from gated SPECT and LVMD impacting CRT response, not forgetting clinical parameters (Figure 1). While each of these parameters have shown in a paper or other its ability to discriminate CRT responder, there is still no single parameter or cutoff value that has high enough diagnostic accuracy and provides robust clinical decision to proceed or amend guidelines. In addition, in the absence of a randomized

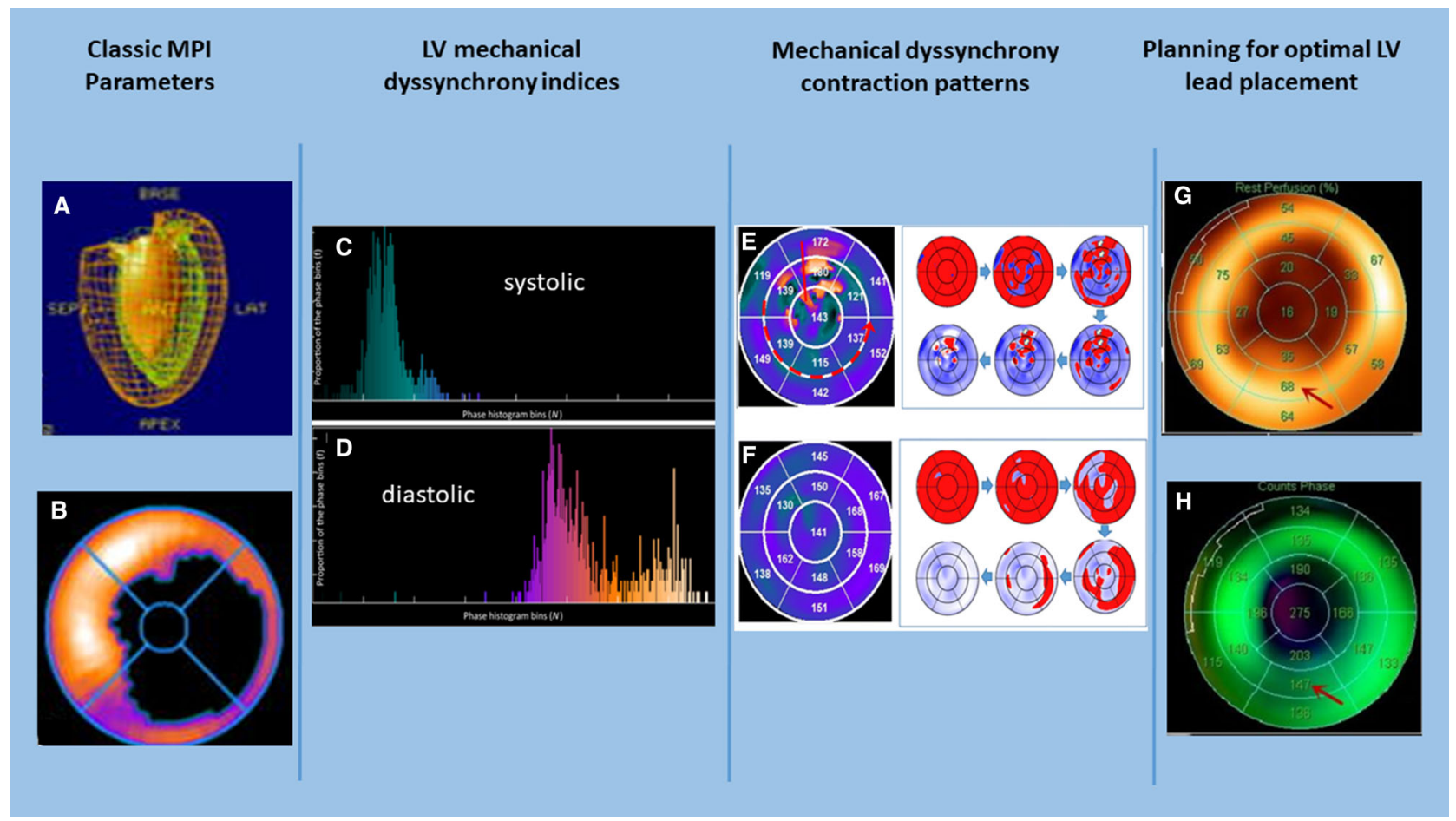

Figure 1. Parameters from gated SPECT MPI that predict and optimize response to CRT. The figure illustrate several parameters extracted from gated myocardial perfusion imaging (MPI) that predict and optimize response to CRT. These include: (1) classical parameters (ejection fractionpanel A, and fixed perfusion defect size-panel B); (2) left ventricular mechanical dyssynchrony indices (i.e. phase standard deviation and bandwidth) from the histogram plots in systolic and diastolic phases (C and D, respectively); (3) contraction patterns (U shape which is more likely to respond to CRT-panel $\mathbf{E}$; and non-U shape which is less likely to respond to CRT-panel $\mathbf{F}$ ); (4) optimal left ventricular (LV) lead placement overlaying the mid inferior myocardium with adequate viability (red arrow-panel G) with the latest onset of mechanical contraction (red arrow-panel $\mathbf{H}$ ), away from large scar burden (dark areas with count less than $50 \%$-panel G) $\mathbf{C}$ and $\mathbf{D}$ are reproduced with permission from Wang et al (Figure 1) ${ }^{23} \mathbf{E}$ and $\mathbf{F}$ are reproduced with permission from Tao et al (Figure 2) ${ }^{18} \mathbf{G}$ and $\mathbf{H}$ are reproduced with permission from Wang et al (Figure 8) ${ }^{17}$. 
Table 1. CRT-response on-line calculator

Female gender: yes/no

Left ventricular end-diastolic volume index: $\mathrm{mL} / \mathrm{m}^{2}$

Left ventricular ejection fraction: \%

Left atrial volume index: $\mathrm{mL} / \mathrm{m}^{2}$

Non-ischemic cardiomyopathy: Yes/No

New York Heart Association class: I-IV

Left bundle branch block: Yes/No

QRS duration: $m s$

Sinus rhythm: Yes/No

Scar burden: \% LV mass

Phase analysis systolic standard deviation:

Phase analysis systolic histogram bandwidth:

$\mathrm{U}$ shape contraction polar map: Yes/No

Phase analysis diastolic standard deviation:

Predicted CRT-response rate: -\%

CRT implantation: recommended; considered; less likely to be helpful

trial, it is hard to make solid changes for the guidelines. However, there might be a solution. The ESC have established an online calculator using more than seven parameters for patients with hypertrophic cardiomyopathy which compiles all data and provides an estimated risk of sudden cardiac death and accordingly gives the recommendation to put an ICD or not. ${ }^{24} \mathrm{We}$ can do a similar online calculator for CRT using MADIT-CRT clinical and echocardiography criteria that predicted best CRT response, adding LVMD phase indices, polar map U shape and scar burden (Table 1). Obviously, these parameters have to be extracted from large multicenter registries, try to identify the most powerful predictors among them and build such model and then validate it. In addition, a certain threshold value for CRT response must be identified to recommend CRT implantation while maintaining cost-effectiveness. This exercise was done for hypertrophic cardiomyopathy, and can be reproduced (although more complex) with CRT.

Perhaps the future of identifying CRT responder is through seeking the help of Radiomics. This is an emerging field of medical study that extracts large quantitative features from medical images using certain data-characteristic algorithm that fail to be identified or appreciated by the naked eye. This has worked with cardiac computed tomography in identifying certain high-risk vulnerable plaques with higher diagnostic accuracy than visual or standard quantitative assessment. ${ }^{25}$ Perhaps radiomics could extract and compiles more data from the perfusion and contraction polar maps through special algorithms and predict better CRT responders. This is an evolving field and showing promises.

Still, many challenges remain to be solved. First, the definition of CRT response using a 5\% change in LVEF and subjective improvement in NYHA class has its limitation; however, it is universally adopted now which simplifies combining data from studies. Second, there are different cut-off values for SD for response; hence a calculator with risk prediction using $\mathrm{SD}$ as continuous variable seems more logical but clinically not helpful to make a decision of yes/no. Third, while most studies published data on LVMD using ECTb quantitative software, many physicians do gated SPECT using other commercially available software with less established data for LVMD. Several studies showed similar values although not identical for LVMD using ECTb vs other software. ${ }^{26,27}$ Efforts have to be made in order to standardize these values, use a phantom to calibrate them to pool large data from multicenters. Fourth, most studies focused on left intraventricular mechanical dyssynchrony and ignored interventricular dyssynchrony (i.e. between right and left ventricles) that plays a crucial role in CRT response. ${ }^{28}$

While this review paper focused on CRT, it also reminded us that LVMD is a powerful prognostic marker of all-cause death, independent of traditional risk factors, and can risk-stratify different cohorts. ${ }^{29,30}$ Moreover, stress induced worsening of LVMD indices has incremental value to diagnose coronary artery disease, similar to LVEF reserve, stunning, and coronary flow reserve. ${ }^{31}$ Therefore, LVMD indices should reported routinely along other gated SPECT data. Combining all these parameters perhaps could yield an objective score that classifies MPI as low, intermediate or high-risk study. Although less data exist with LVMD and PET, these parameters were also tested with gated $\mathrm{PET}^{32}$ and should be further be studied and reported, particularly that the field of PET is evolving at a very fast pace.

\section{Disclaimers}

Patients with life expectancy less than 6 months are excluded If CRT implantation is recommended, LV lead should be placed at latest site of onset mechanical contraction and away from high scan burden area to ensure high rate of CRT response.

\section{Disclosure}

The authors have indicated that they have no financial conflict of interest. 


\section{References}

1. Bristow MR, Saxon LA, Boehmer J, Krueger S, Kass DA, De Marco T, et al. Cardiac-resynchronization therapy with or without an implantable defibrillator in advanced chronic heart failure. $\mathrm{N}$ Engl J Med 2004;350:2140-50.

2. Cleland JG, Calvert MJ, Verboven Y, Freemantle N. Effects of cardiac resynchronization therapy on long-term quality of life: An analysis from the cardiac resynchronisation-heart failure (care-hf) study. Am Heart J 2009;157:457-66.

3. Cleland JG, Daubert JC, Erdmann E, Freemantle N, Gras D, Kappenberger L, et al. The effect of cardiac resynchronization on morbidity and mortality in heart failure. $\mathrm{N}$ Engl $\mathrm{J}$ Med 2005;352:1539-49.

4. Epstein AE, DiMarco JP, Ellenbogen KA, Estes NA 3rd, Freedman RA, Gettes LS, et al 2012 accf/aha/hrs focused update incorporated into the accf/aha/hrs 2008 guidelines for devicebased therapy of cardiac rhythm abnormalities: A report of the american college of cardiology foundation/american heart association task force on practice guidelines and the heart rhythm society. Circulation 2013;127:e283-352.

5. Goldenberg I, Moss AJ, Hall WJ, Foster E, Goldberger JJ, Santucci $\mathrm{P}$, et al. Predictors of response to cardiac resynchronization therapy in the multicenter automatic defibrillator implantation trial with cardiac resynchronization therapy (madit-crt). Circulation 2011;124:1527-36.

6. Bax JJ, Abraham T, Barold SS, Breithardt OA, Fung JW, Garrigue $\mathrm{S}$, et al. Cardiac resynchronization therapy: Part 1-issues before device implantation. J Am Coll Cardiol 2005;46:2153-67.

7. Bertini M, Hoke U, van Bommel RJ, Ng AC, Shanks M, Nucifora $\mathrm{G}$, et al. Impact of clinical and echocardiographic response to cardiac resynchronization therapy on long-term survival. Eur Heart J Cardiovasc Imaging 2013;14:774-81.

8. Chung ES, Leon AR, Tavazzi L, Sun JP, Nihoyannopoulos P, Merlino J, et al. Results of the predictors of response to crt (prospect) trial. Circulation 2008;117:2608-16.

9. AlJaroudi W, Chen J, Jaber WA, Lloyd SG, Cerqueira MD, Marwick T. Nonechocardiographic imaging in evaluation for cardiac resynchronization therapy. Circ Cardiovasc Imaging 2011;4:334-43.

10. Chen J, Garcia EV, Folks RD, Cooke CD, Faber TL, Tauxe EL, et al. Onset of left ventricular mechanical contraction as determined by phase analysis of ecg-gated myocardial perfusion spect imaging: Development of a diagnostic tool for assessment of cardiac mechanical dyssynchrony. J Nucl Cardiol 2005;12:687-95.

11. Lin X, Xu H, Zhao X, Folks RD, Garcia EV, Soman P, et al. Repeatability of left ventricular dyssynchrony and function parameters in serial gated myocardial perfusion spect studies. J Nucl Cardiol 2010;17:811-6.

12. Abraham T, Kass D, Tonti G, Tomassoni GF, Abraham WT, Bax JJ, et al. Imaging cardiac resynchronization therapy. JACC Cardiovasc Imaging 2009;2:486-97.

13. Henneman MM, Chen J, Dibbets-Schneider P, Stokkel MP, Bleeker GB, Ypenburg C, et al. Can lv dyssynchrony as assessed with phase analysis on gated myocardial perfusion spect predict response to crt? J Nucl Med 2007;48:1104-11.

14. Boogers MM, Van Kriekinge SD, Henneman MM, Ypenburg C, Van Bommel RJ, Boersma E, et al. Quantitative gated spectderived phase analysis on gated myocardial perfusion spect detects left ventricular dyssynchrony and predicts response to cardiac resynchronization therapy. J Nucl Med 2009;50:718-25.

15. Adelstein EC, Saba S. Scar burden by myocardial perfusion imaging predicts echocardiographic response to cardiac resynchronization therapy in ischemic cardiomyopathy. Am Heart J 2007;153:105-12.
16. Friehling M, Chen J, Saba S, Bazaz R, Schwartzman D, Adelstein $\mathrm{EC}$, et al. A prospective pilot study to evaluate the relationship between acute change in left ventricular synchrony after cardiac resynchronization therapy and patient outcome using a single-injection gated spect protocol. Circ Cardiovasc Imaging 2011;4:5329.

17. Wang J, Wang Y, Yang M, Shao S, Tian Y, Shao X, et al. Mechanical contraction to guide crt left-ventricular lead placement instead of electrical activation in myocardial infarction with left ventricular dysfunction: An experimental study based on non-invasive gated myocardial perfusion imaging and invasive electroanatomic mapping. J Nucl Cardiol 2019

18. Tao N, Qiu Y, Tang H, Qian Z, Wu H, Zhu R, et al. Assessment of left ventricular contraction patterns using gated spect mpi to predict cardiac resynchronization therapy response. J Nucl Cardiol 2018;25:2029-38.

19. Fudim M, Daalgart F, Fathallah M, Iskandrian AE, Borges-Neto S. Mechanical dyssynchrony: How do we measure it, what it means, and what we can do about it. J Nucl Cardiol 2019

20. Aljaroudi W, Alraies MC, Halley C, Rodriguez L, Grimm RA, Thomas JD, et al. Impact of progression of diastolic dysfunction on mortality in patients with normal ejection fraction. Circulation 2012;125:782-8.

21. Hsu TH, Huang WS, Chen CC, Hung GU, Chen TC, Kao CH, et al. Left ventricular systolic and diastolic dyssynchrony assessed by phase analysis of gated spect myocardial perfusion imaging: A comparison with speckle tracking echocardiography. Ann Nucl Med 2013;27:764-71.

22. Fudim M, Fathallah M, Shaw LK, Liu PR, James O, Samad Z, et al. The prognostic value of diastolic and systolic mechanical left ventricular dyssynchrony among patients with coronary heart disease. JACC Cardiovasc Imaging 2018

23. Wang C, Tang H, Zhu F, Jiang Z, Shi J, Zhou Y, et al. Prognostic value of left-ventricular systolic and diastolic dyssynchrony measured from gated spect mpi in patients with dilated cardiomyopathy. J Nucl Cardiol 2018

24. O'Mahony C, Jichi F, Pavlou M, Monserrat L, Anastasakis A, Rapezzi C, et al. A novel clinical risk prediction model for sudden cardiac death in hypertrophic cardiomyopathy (hcm risk-scd). Eur Heart J 2014;35:2010-20.

25. Kolossvary M, Park J, Bang JI, Zhang J, Lee JM, Paeng JC, et al. Identification of invasive and radionuclide imaging markers of coronary plaque vulnerability using radiomic analysis of coronary computed tomography angiography. Eur Heart J Cardiovasc Imaging 2019

26. AlJaroudi W, Jaber WA, Grimm RA, Marwick T, Cerqueira MD. Alternative methods for the assessment of mechanical dyssynchrony using phase analysis of gated single photon emission computed tomography myocardial perfusion imaging. Int J Cardiovasc Imaging 2012;28:1385-94.

27. Okuda K, Nakajima K, Matsuo S, Kashiwaya S, Yoneyama H, Shibutani T, et al. Comparison of diagnostic performance of four software packages for phase dyssynchrony analysis in gated myocardial perfusion spect. EJNMMI Res 2017;7:27.

28. Zhou W, Jiang Z, Chen J, Garcia EV, Li D. Development and validation of a phase analysis tool to measure interventricular mechanical dyssynchrony from gated spect mpi. J Nucl Cardiol 2017;24:1680-6.

29. Hess PL, Shaw LK, Fudim M, Iskandrian AE, Borges-Neto S. The prognostic value of mechanical left ventricular dyssynchrony defined by phase analysis from gated single-photon emission computed tomography myocardial perfusion imaging among patients with coronary heart disease. J Nucl Cardiol 2017;24:48290. 
30. Mori H, Isobe S, Suzuki S, Unno K, Morimoto R, Kano N, et al. Prognostic value of left ventricular dyssynchrony evaluated by gated myocardial perfusion imaging in patients with chronic kidney disease and normal perfusion defect scores. J Nucl Cardiol 2019;26:288-97.

31. Jiang Z, Tang H, Shi J, Zhou Y, Wang C, Li D, et al. Myocardial stunning-induced left ventricular dyssynchrony on gated singlephoton emission computed tomography myocardial perfusion imaging. Nucl Med Commun 2018;39:725-31.
32. AlJaroudi W, Alraies MC, Menon V, Brunken RC, Cerqueira MD, Jaber WA. Predictors and incremental prognostic value of left ventricular mechanical dyssynchrony response during stress-gated positron emission tomography in patients with ischemic cardiomyopathy. J Nucl Cardiol 2012;19:958-69.

Publisher's Note Springer Nature remains neutral with regard to jurisdictional claims in published maps and institutional affiliations. 